\title{
EL PLANCTON DEL ATLANTICO SUROESTE. DINAMICA Y ECOLOGIA
}

\author{
ANGELES ALVARIÑO \\ 7535 Cabrillo Avenue - La Jolla, California 92037 - U.S.A.
}

Plankton was collected in Nov.- Dec. 1972, during the Cato-6 Expedition ( $R / V$ Melville). Distribution of Siphonophores and Medusap and ecological mplications pointed out by these organisms are discussed. 25 species of Siphonopnorae were obtained corresponding to tropical. temperate, and Antarctic-Subantarctic categories. 5 species of Medusae appeared, showing the peculiar erratic distribution typical of these organisms. Faunistic data are presented in tables and maps.Effects of Brazil, Falkland Currents and upwelling, centers appear well defined by the distribution of the siphonophores. Cosmopolitan siphonophores, typical of temperate and warm waters inhabited a band (zone of Rio Grande rise) between the southernmost and northernmost progressions of Brazil and Falkland Currents. These populations are carried to. ward Southamerican coasts by the anticyclonic circulation of the Central South Atlantic, forming a convergence; and a divergence towards the north is responsible for development of upwelling systems.

\section{Introducción}

Se analiza el plancton de la región meridional de la Corriente de Brasil y la zona más al sur, según aparece en la Tabla I. Se discute la distribución de sifonóforos y medusas en relación con las especies de quetognatos indicadores típicos de condiciones oceánicas.

Estudios previos sobre sifonóforos en esta región se incluyen en Alvariño $(1971,197$ ), y las medusas aparecen recopiladas en Kramp (1959, 1961).

Esta región se encuentra bajo la influencia de la Corriente de Brasil, extensión de la Corriente de Malvinas y el Atlántico Central meridional, cuyas aguas avanzan desde el Este hacia el continente americano, formando una barrera entre las dos Corrientes.

Los datos hidrográficos obtenidos se discuten en Reid. Nowlin \& Patzert (1977), pero estos autores, asi como Buscaglia (1971) se ocupan de la circulación profunda.

\section{Materiales y Métodos}

Las colecciones fueron obtenidas en Nov.-Dic. 1972, por e) buque oceanográfico Melville, durante la Expedición Cato-6. Se empleó una red de $1 \mathrm{~m}$ de diametro en la boca y malla de 500 micras, en arrastre oblíquo desde $180 \mathrm{~m}$ de profundidad o menos nasta la superfície Tabla 1 . Se desconece la cantidad de agua filtrada en cada arrastre y se han podido normalizar los va'ores cuantitativos. Así, el número de individuos en la Tabla II, corresponde al contaje directo de la totalidad de la muestra para cada localidad, sin tomar porciones alícuotas.

En los mapas se indican las estaciones ocupadas y la presencia o ausencia de las especies.

\section{La distribución de los Sifonóforos}

Las aguas de la Corriente de Brasil fluyen hacia el sur frente al continente, hasta aproximadamente $38^{\circ} \mathrm{S}$, y la rama más oceánica diverge hacia el Este. Las poblaciones arrastradas desaparecen parcial o totalmente con la dispersión de estas aguas en la región templada. El gran remolino de giro anticiciónico (en contra de las agujas del reloj) transporta en su avance hacia el Oeste, poblaciones de la región templada de la zona Central del Atlántico Sur. La Corriente de Malvinas avanza hacia los $40^{\circ} \mathrm{S}$. extendiéndose hacia el Este para integrarse en el remolino anti. ciclónico Subtropical.

La distribución de los sifonóforos señala fronteras bio. lógicas que delimitan la extensión e influencia de las aguas oceánicas de distintas características físico-químicas y bióticas.

En estos estudios se han observado 25 especies de sitonoforos, la mayoría de régimen tropical, varias cosmopolitas (habitantes de regiones templadas y cálidas) $\mathrm{v}$ otras típicas de las regiones Antártica y Subantártica. Los sitonóforos observados han sido previamente determinados en esta región (Alvariño, 1971) a excepción de Abyla carina, Epibulia ritteriana y Lilyopsis rosea. Sin embargo, otras 30 especies observadas por distintos autores (Alvariño, 1972) no aparecían en estas colecciones. Esta escasez se debe a restricciones del muestreo que abarca 17 dias, y la particularidad observada (Alvarino, 1977) en las meausas. que puede aplicarse a los sifonóforos. Así, un numero reducido de especie: aparecen frecuente $\mathrm{y}$ abundantemente, $\mathrm{y}$ las demás son raras, de caracter esporádico y errátil. En el caso de poblaciones aglomeradas, poco dispersas, tendría que coincidir el muestreo exactamente con tales concentraciones en el oceáno. tanto en tiempo como en espacio, para determinar su presencia

Además no se han encontrado las especies neríticas. $M$. atlantica, M. bargmannae. $M$. kochi, cuya distribución seria interesante conocer en esta región, y Ch. contorta, especie tropical, previamente observada en esta zona (Alvariño, 1971).

La influencia tropical marcada por la Corriente de Brasil aparece determinada por las especies trovicales, $D$. bojani. $L$. ros. sack, S. chuni, S. monoica, S.turgida, A. eschscholtzi, y el quetognato Sagitta helenae (Figs. 1 a 5). Indicios de un avance débil de estas aguas y las reliquias aue persisten de una progresión anterior, se identiticam $C$. sagittata, $A$. cartna, $E$. hyalinum, $B$. bassensis y $E$. richardi. En esta categoria hay que incluir la población remanente de $D$. mitra, en una localidad bajo la influencia Subantártica (Figs. 2, 6, 7, 8).

Hay que destacar la distribución de $D$. bojani y $A$. eschs. choltzi señalando el efecto de ias aguas del Atlántico Central (Figs. $1,4)$ en su giro anticiclónico hacia Suramérica. La distribución de $D$. bojani se interrumpe en esta banda de convergeneia, avanzando hacia el Sur con la Corriente costera. En cambio A. eschs. choltzi se extiende al norte y sur de la zona de convergencia.

$E$. spiralis y $A$. tetragona que en otras regiones son indicadoras de la influencia tropical, en esta zona coinciden con el avance de las aguas templadas del Atlántico Sur Central (Figs. 9, 10), aún cuando la última especie conserva características de la influencia cálida.

La convergencia de giro anticiclónico aparece definida por especies de régimen templado, Ch. appendiculata, D. dispar, C. leuckarti, A. ernesti, H. hippopus, Ch. multidentata (Figs. 8, 11 a 14). E. ritteriana problablemente con prefexencia por aguas calidas, ocurtía en la confluencia el giro antíciclónico y el avance Subantártico (Fig. 13).

La influencia Antártica y Subentártica esta definida por $M$. antarcticus, $D$ arctica, $L$, handy, $L$, rosea $y$ el quetognato Sagitta gazellae (Figs. $8,15,16,5$ ). D. arctica se extiende del Artico al Antártico en el Atlántico y Pacífico, avanzando también por el Océano Indico, y su presencia en los estratos del epiplancton ocurre en zonas bajo la influencia de las corrientes polares o en surgencias. Su presencia en la Estacion 42 señala el avance de la rama oriental de la Corriente de Malvinas.

Un núcleo de surgencia aparece en la zona de divergencia de las aguas de Brasil (Estacion $33,25^{\circ} 16^{\prime} \mathrm{S}-40^{\circ} 01 \mathrm{~W}^{\prime}$ ) señalado por el quetognato mesopelágico $S$. zetesios en el epiplancton (Fig. 5). En esta localidad se observaron también los quetognatos $S$. bipunctata. S. hexaptera. S. serratodentata. Pternsagitta draco. Krohnitta subtilis, y la medusa Eutima gracilis, indicando una fuerte confluencia faunística.

Los sifonóforos más frecuentes en estas colecciones fueron Ch. appenciculata e H. hippopus.

\section{Distribución de las Medusas}

Solamente se han encontrado cinco especies: $R h$. velatum, $E$. gracilis, $H$. schultzei, $P$. clara, $C$. papillata. La especia más abundante, $R h$. velatum es tambien una de las más frecuentes en otra: localidades oceánicas. P. clara, H. schultzei, C. papillata no han sido observadas previamente en esta región, pero habitan las aguas africanas adyacentes (Figs. 17. 18). La distribución de las medu sas es esporádicà, según se ha mencionadc, y únicamente las especies holoplanctónicas (Traquimedusas y Narcomedusas) aparecen con mayor frecuencia que las demas especies de régimen meroplanctónico. 
TABLA I

DISTRIBUCION DE LAS COLECCIONES ESTUDIADAS

EXPEDICION CATO-6, BUQUE OCEANOGRAFICO MELVILLE

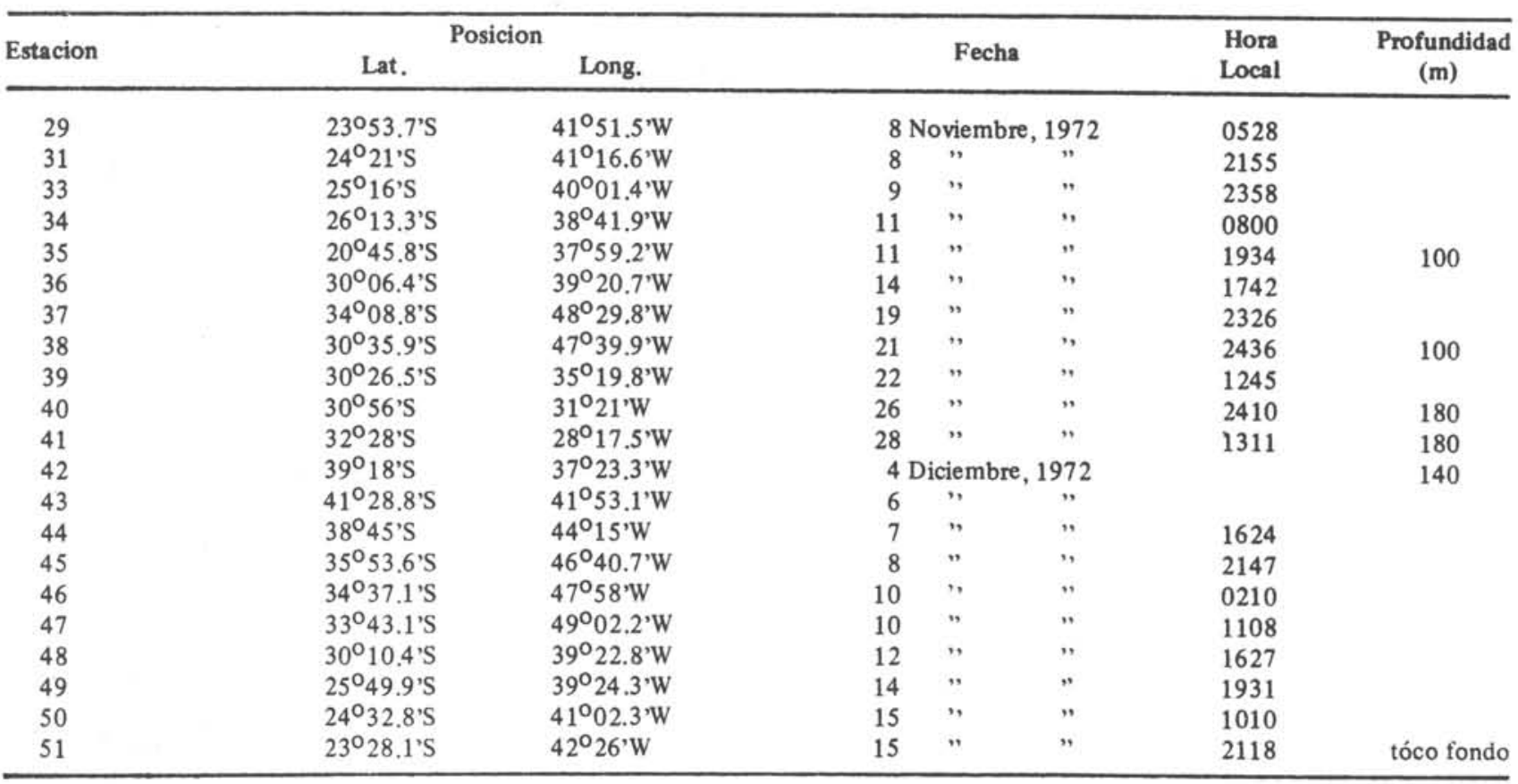

TABLA II

DISTRIBUCION DE LOS SIFONOFOROS EN EL ATLANTICO SUROESTE.

EXPEDICION CATO-6, NOVIEMBRE-DECIEMBRE, 1972.

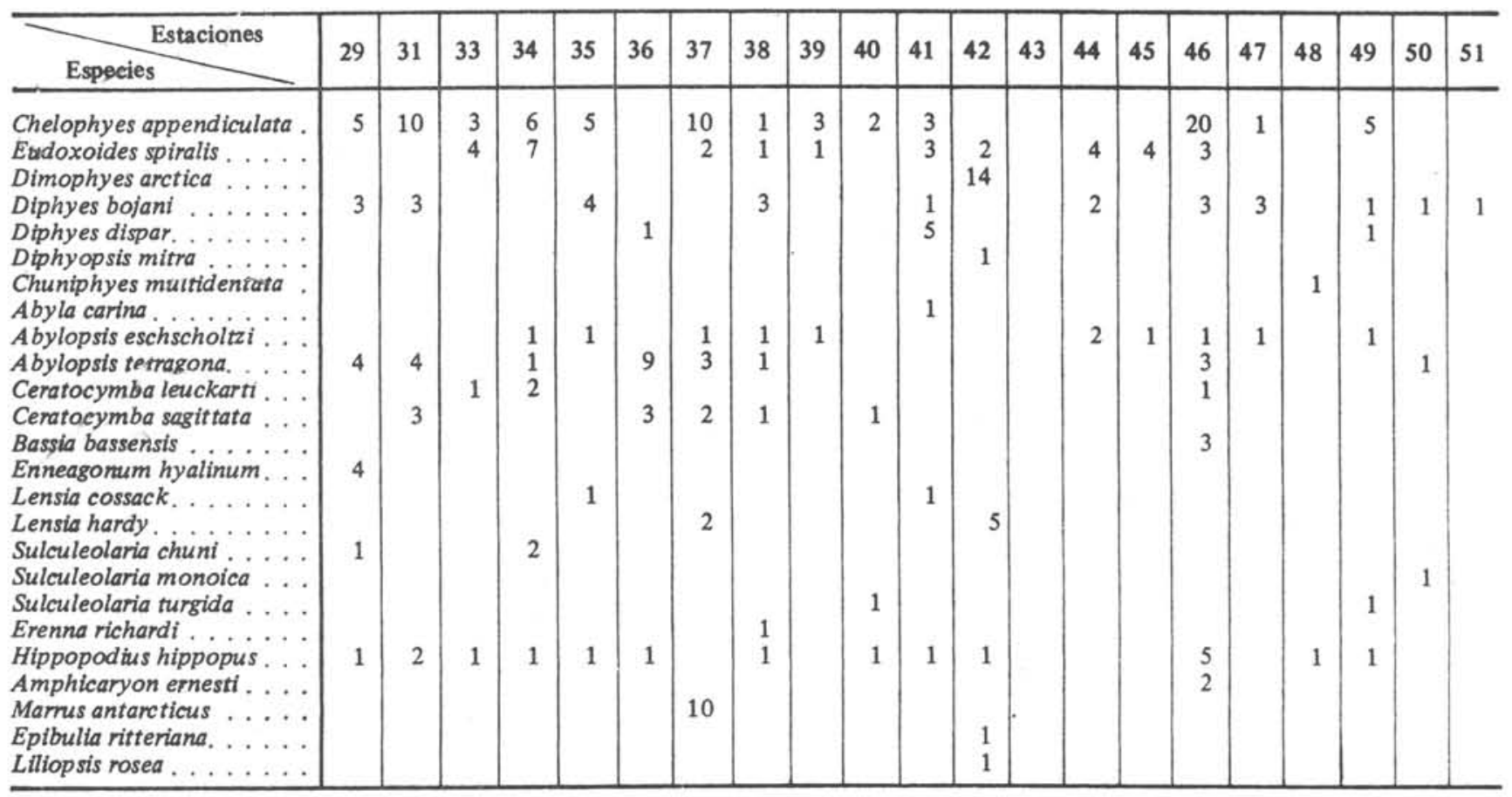



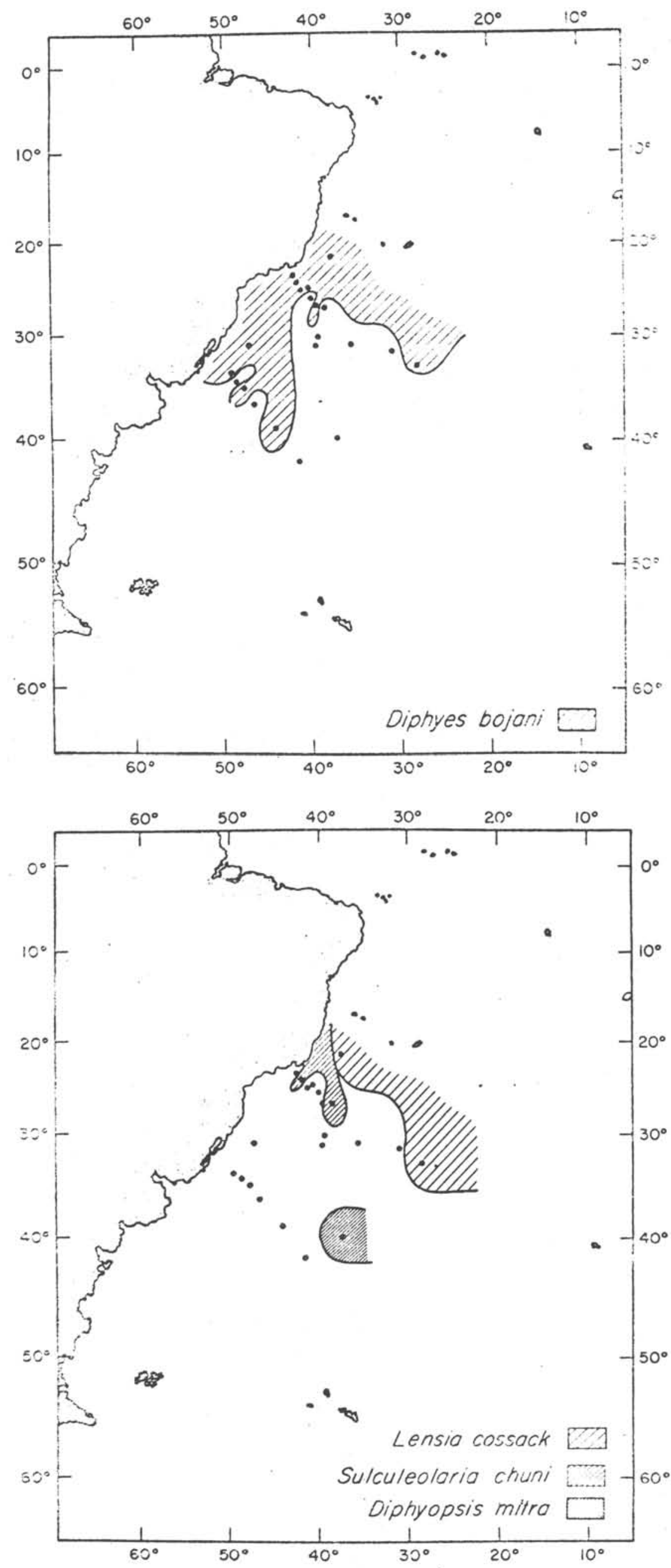

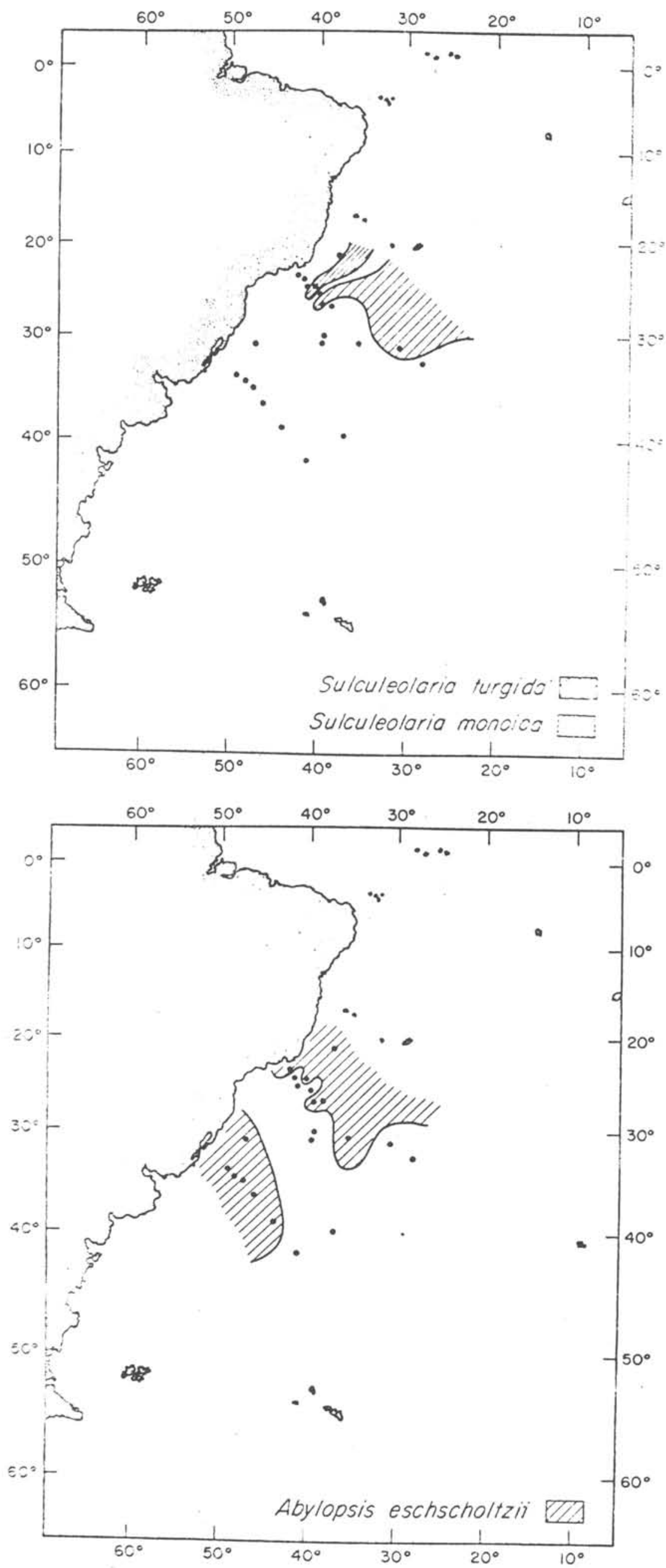

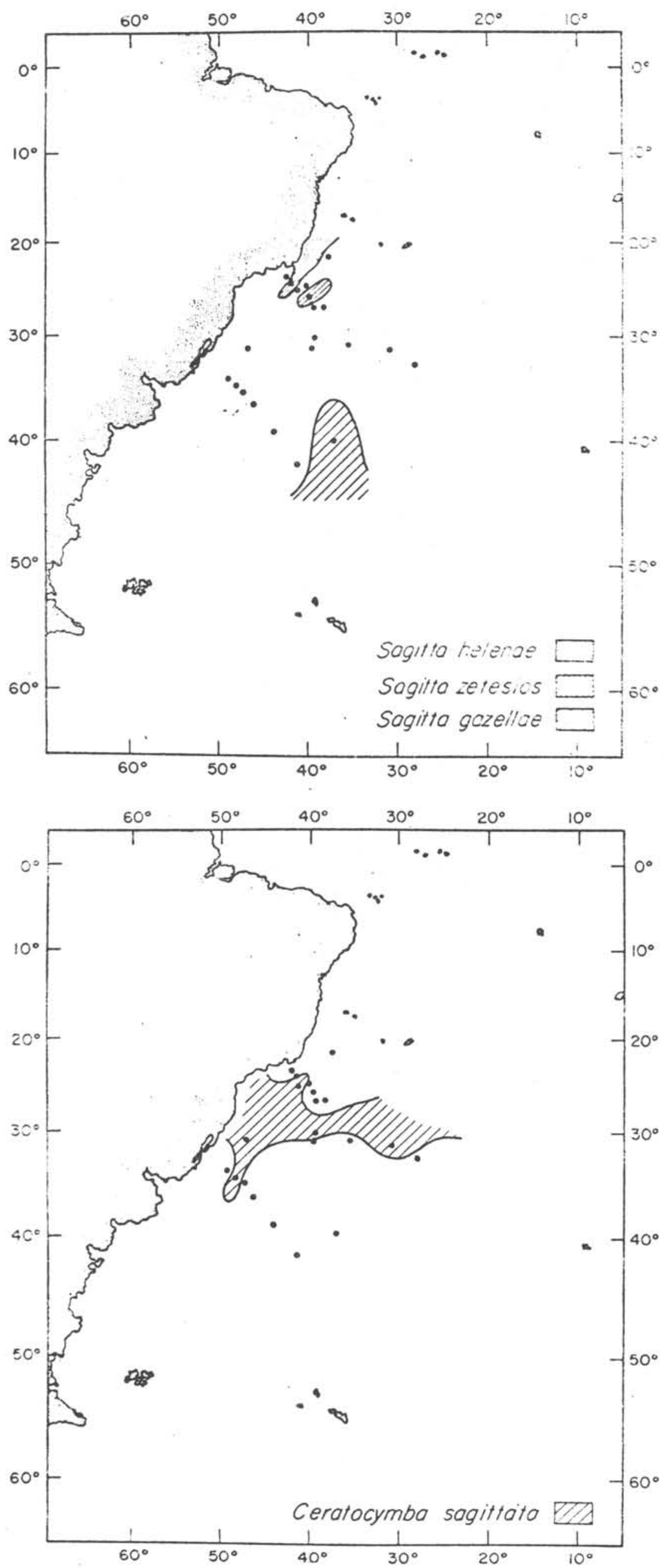

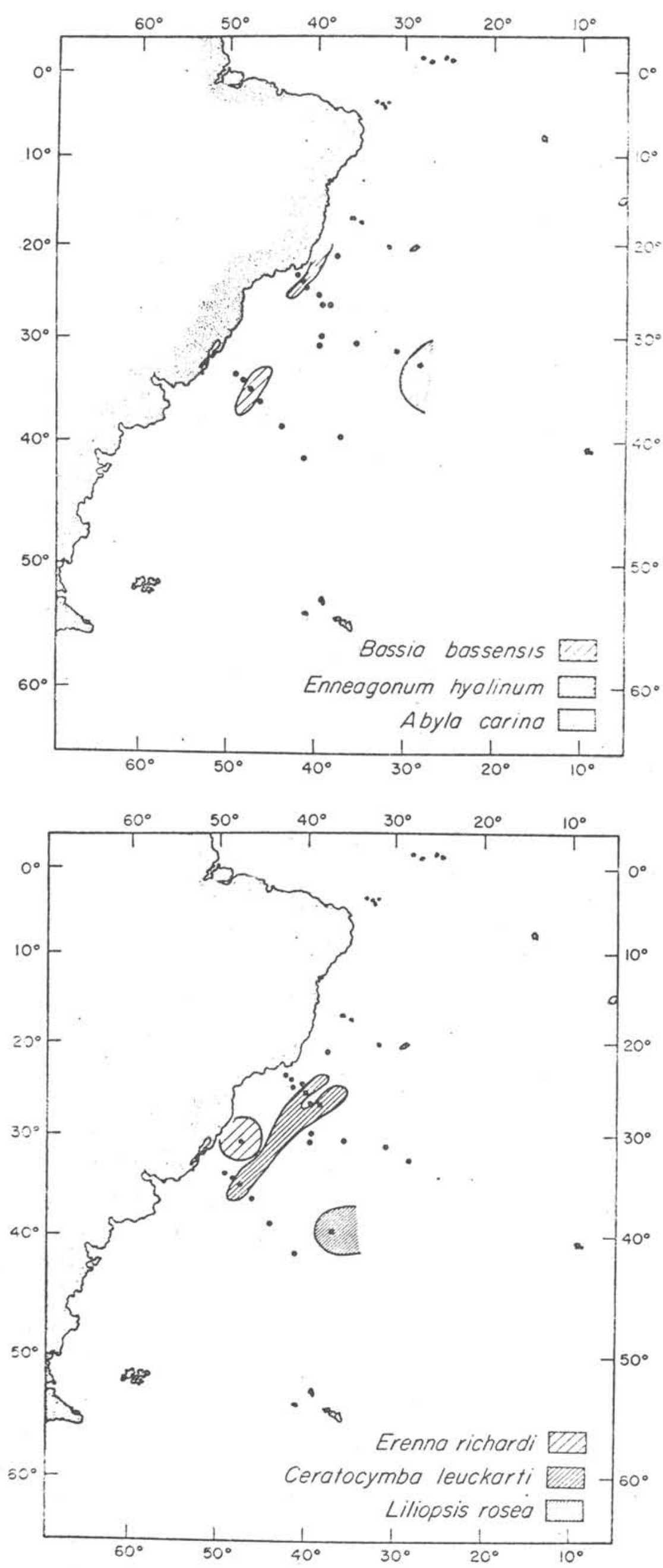

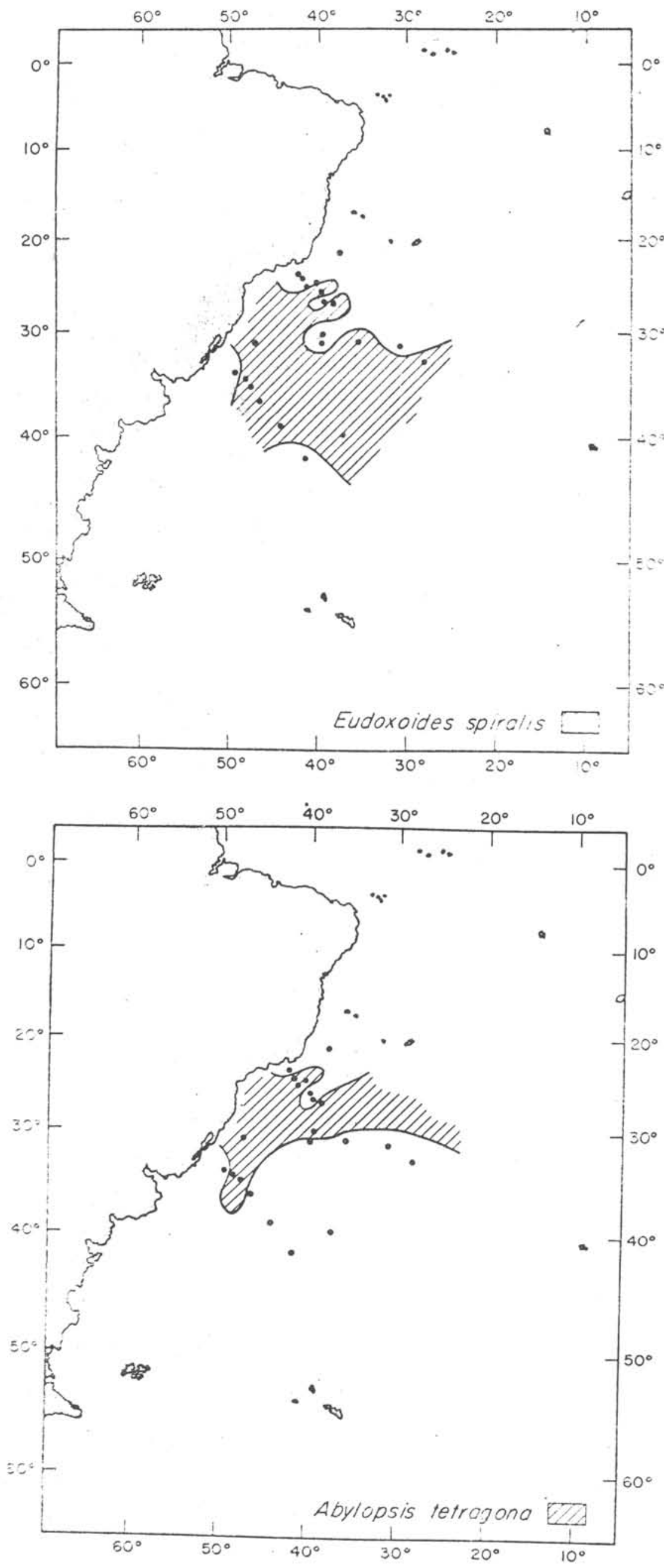

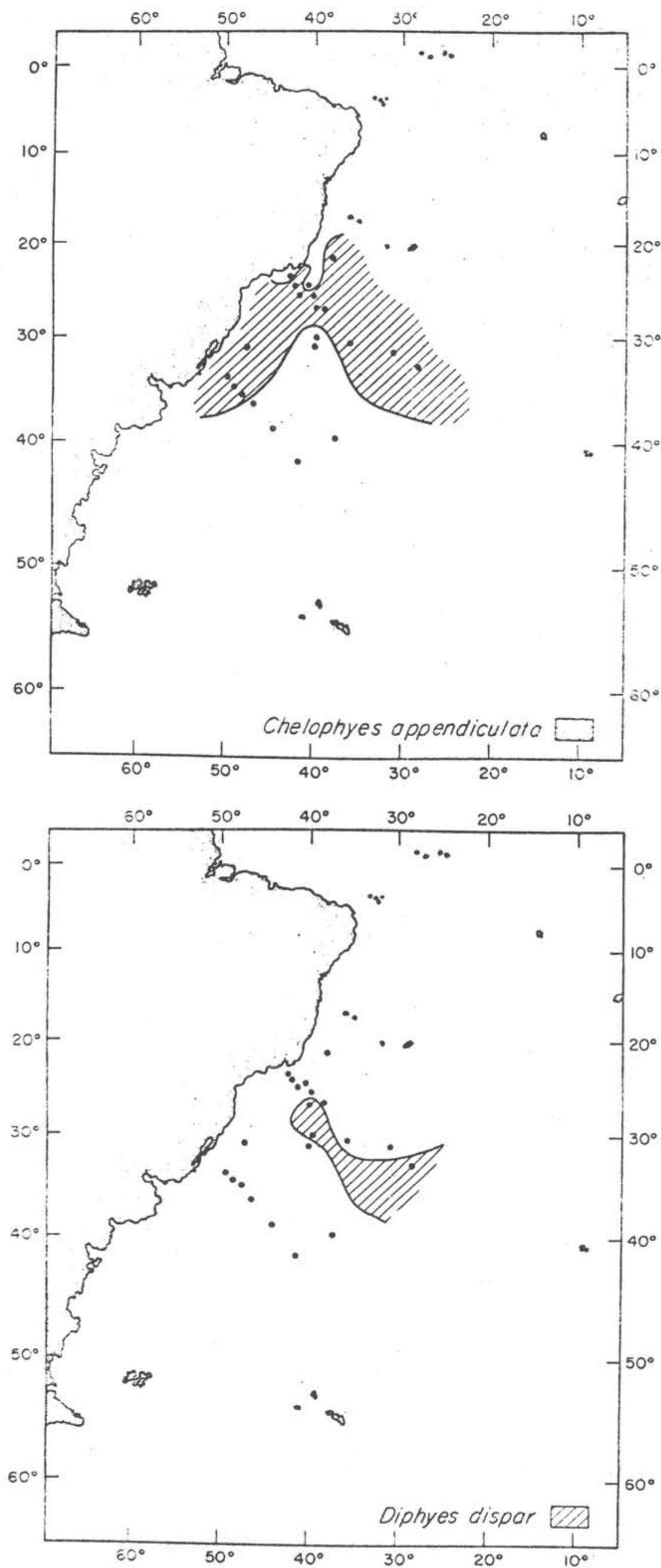

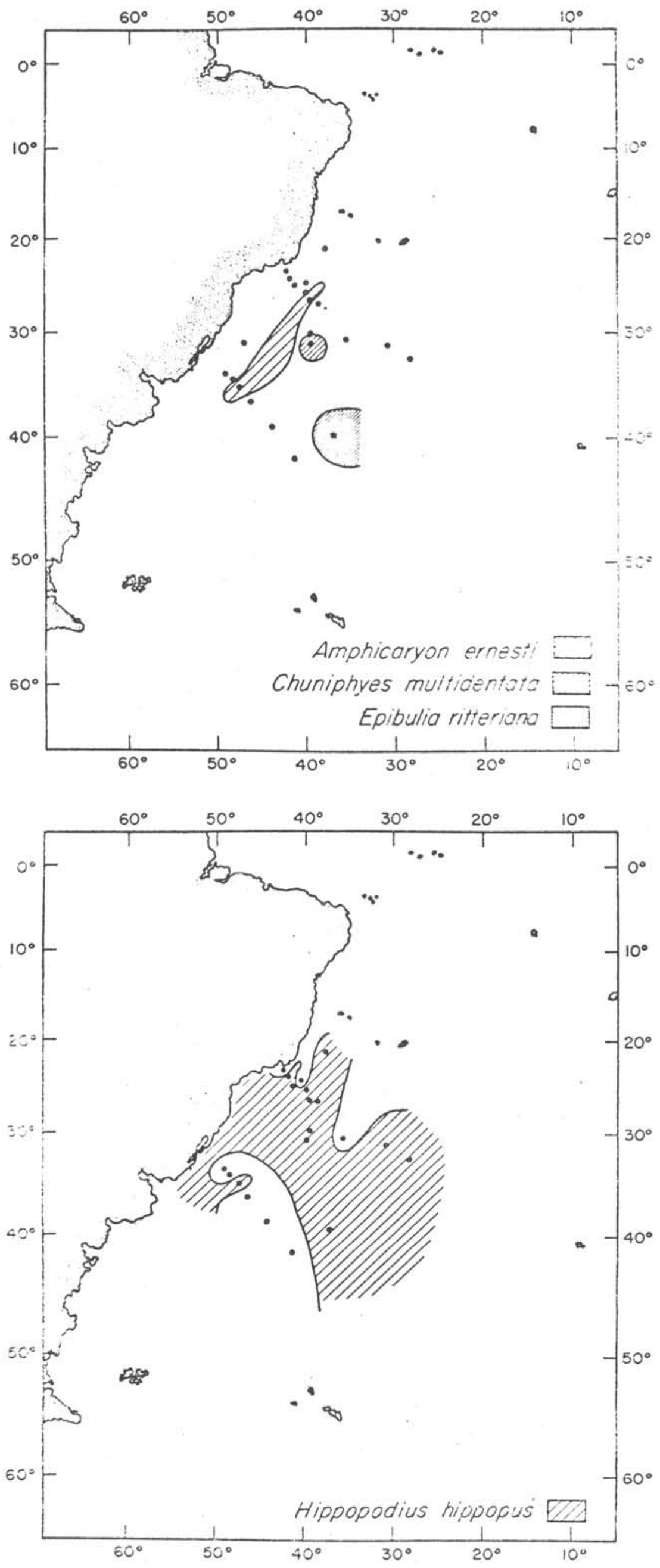

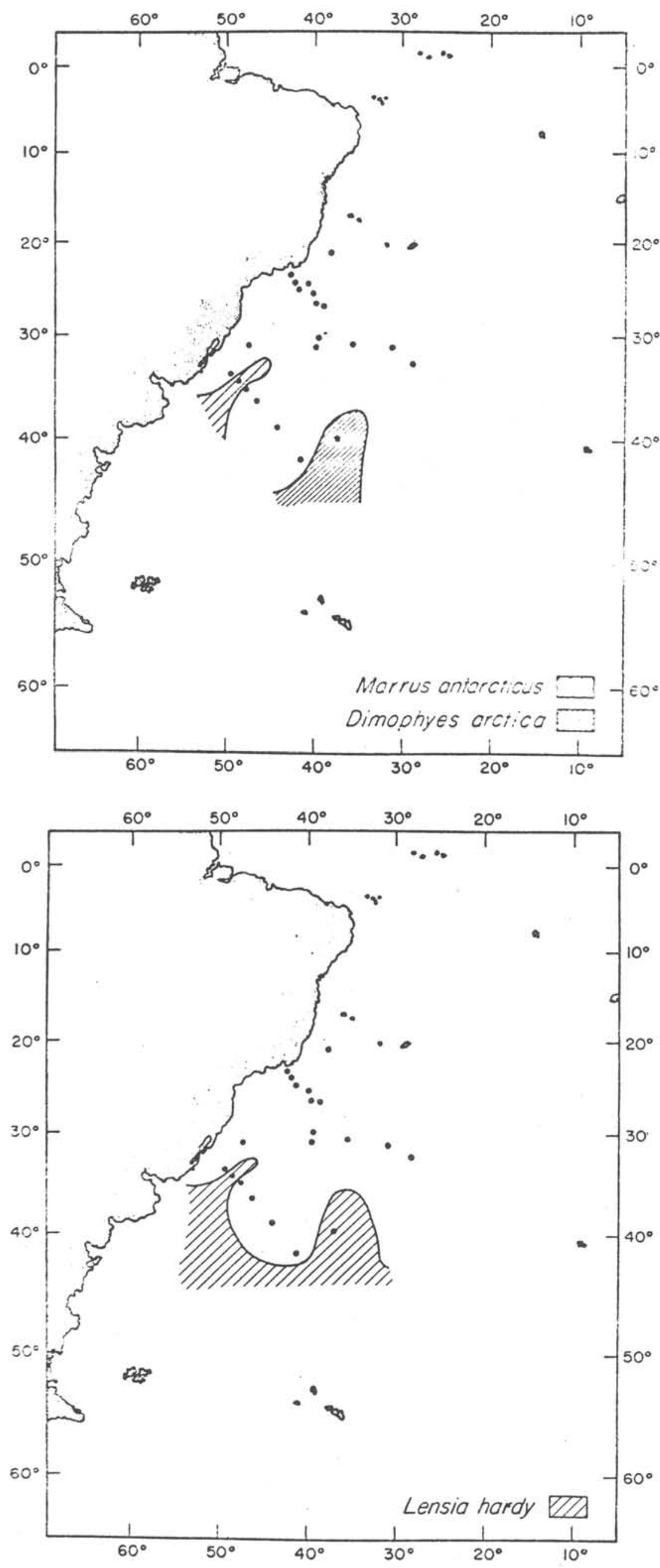

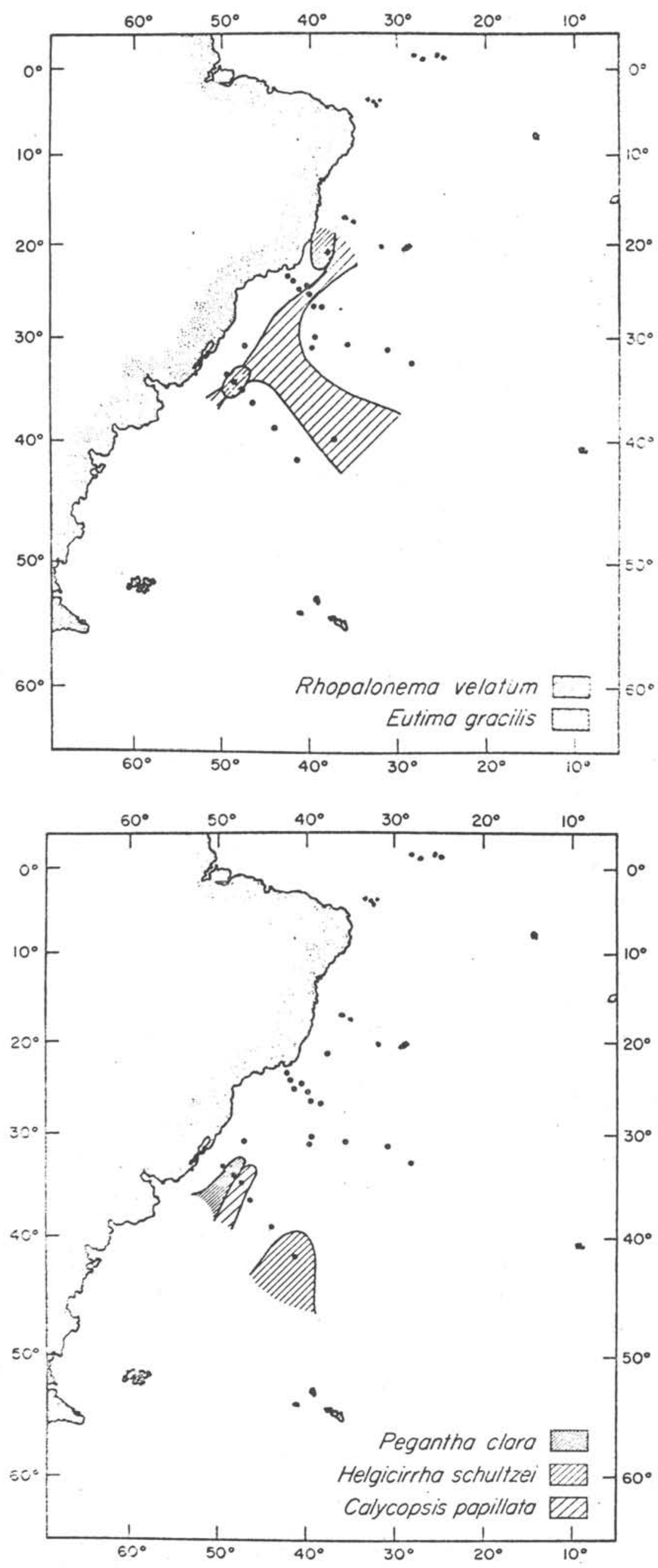


\section{Consideraciones finales}

Se precisa un estudio continuado en esta región para determinar la extensión e intensidad de la influencia tropical, régimen anticiclónico del Atlántico Central. la corriente costera cálida, la progresión de las aguas Antártico-Subantárticas y el sistema de surgencias. Se obtendrán resultados definitivos con el estudio del plancton, base necessaria para establecer programas de oceanografía y pesca. Notaciones de salinidad y temperatura no son suficientes para clasificar las aguas, pero el complejo planctónico define con precisión las regiones oceánicas. Así mismo es importante desarrollar conjuntamente estudios de las poblaciones neríticas, bentónicas y nectónicas en esta región. Variaciones en la estructura dinámica del oceano, repercuten en las características de las poblaciones pesqueras. La puesta de huevos y supervivencia de larvas y jóvenes está controlada por factores abióticos y en mayor extensión por los bióticos, y la resultante determinará las poblaciones de reclutas que se integran a los cardúmenes pesqueros.

\section{Refẹrencias Bïbliográficas}

ALVARIÑO, A. 1971. Siphonophores of the Pacific with a review of the world distribution. Bull. Scripps Instn Oceanogr., (16): $1-432$

- _- 1972. A second record of a rare siphonophore Epibulia ritteriana Haeckel, 1888. Fish. Bull. NOAA, 70(2): 507-509.

-- - - 1977. Depredadores planctónicos y la pesca. Mems II Simn. Latinoam. Oceanorr. Biol., 1:141-160. (en preparación).

BUSCAGLIA, J.L. 1971. On the curculation of the intermediate water in the South western Atlantic Ocean. J. mar. Res., $29: 245-255$

KRAMP, P. L. 1959. The Hydromedusae of the Atlantic Ocean and adjacent waters. Dana Rep., (46):1-283.

mar. Biol. Ass. U. K., 40:1-469.

REID, J. L.; NOWLIN, W. D. \& PATZERT, W. C. 1977 . On the characteristics and circulation of the Southwestern Atlantic Ocean. J. phys. Oceanogx., 7(1) :61-91. 